\title{
A Unified Approach to the Finite-Horizon Linear Quadratic Optimal Control Problem*
}

\author{
Augusto Ferrante ${ }^{1}$ and Lorenzo Ntogramatzidis ${ }^{2, * *}$ \\ ${ }^{1}$ Dipartimento di Ingegneria dell' Informazione Università di Padova, via Gradenigo, 6/B-35131 Padova, Italy; ${ }^{2}$ Department of \\ Electrical and Electronic Engineering The University of Melbourne, Parkville, VIC 3010, Australia
}

Under the mild assumption of sign-controllability, a closed-form expression parameterizing all the solutions of the Hamiltonian differential equation over a finite time interval is presented in terms of a strongly unmixed solution of an algebraic Riccati equation ( $A R E$ ) and of the solution of an algebraic Lyapunov equation. This result is employed for the solution of a generalized version of the finite-horizon linear quadratic (LQ) problem, encompassing the case of fixed end-point. Furthermore, it is shown how this method can be applied to the $\mathrm{H}_{2}$ preview decoupling problem.

Keywords: Algebraic Riccati equation; differential Riccati equation; $H_{2}$ decoupling with preview; signcontrollability; strongly unmixed solutions of an ARE

\section{Introduction}

The linear quadratic (LQ) regulator is the prototype of a large variety of fundamental optimization problems in system and control theory, and for this reason has always received a great deal of attention. In this paper, we refer to the finite-horizon LQ problem, whose solution is given in the literature in terms of the solution of a Riccati differential equation, $[2,18]$. This paper proposes a different perspective to deal with this

*Partially supported by the Ministry of Higher Education of Italy (MIUR) under the project Identification and Control of Industrial Systems any by the Australian Research Council (DP0664789).

${ }^{* *}$ Correspondence to: L. Ntogramatzidis, E-mail: Int@ee.unimelb. edu.au problem and presents a new methodology for its solution. Such a methodology does not require the integration of the Riccati differential equation and provides the solutions of different versions of the LQ problem in a unified framework. In particular, the problem statement considered encompasses the standard case of assigned initial state and quadratically weighted terminal state as well as that of both assigned initial and terminal states (the latter usually being referred to as the fixed end-point LQ, [6,18,23]). Indeed, differently from the existing techniques, this methodology applies also to LQ problems with (i) affine constraints on the states at the end-points and (ii) a performance index including an arbitrary positive semidefinite quadratic penalization term weighting the difference between the states at the end-points and two assigned target states.

The proposed method is based on a closed-form formula parameterizing the set of trajectories solving the Hamiltonian differential equation. From such trajectories, the optimal solution is selected by imposing the suitable boundary conditions. Different parameterizations of the solutions of the Hamiltonian differential equation have been presented in [13] and [20] for the solution of finite-horizon LQ problems and $\mathrm{H}_{2}$ preview problems. In particular, in [13] such parameterization involves the stabilizing and antistabilizing solution of the infinite-horizon algebraic Riccati equation (ARE), hence requiring the assumption of controllability of the underlying system. In [20], the reachability canonical decomposition is exploited

Received 5 December 2006; Accepted 27 July 2007

Recommended by J.C. Geromel, A.S. Morse 
to derive an alternative parameterization under the milder assumption of stabilizability. In this paper, the material in [13] and [20] is generalized in several directions. In fact, we only need the sign-controllability of the underlying system, $[26,16,25]$, which is an extremely weak system theoretic assumption. Furthermore, contrarily to [20], we avoid the employment of a state space decomposition, which is not robust from a computational viewpoint. Indeed, the parameterized expression of the solutions of the Hamiltonian differential equation herein presented involves the unmixed solution of an ARE, [26,16,25], and the solution of a Lyapunov equation. Finally, as already observed, the optimal control problem analyzed and solved herein is more general than those presented in $[13,20]$.

The advantages of the present approach are:

- Nonstandard LQ optimization problems, that cannot be handled with the existing tools of $L Q$ theory, can be explicitly solved by exploiting the material herein developed. A specific example will be discussed in Section 3.1.

- We derive closed-form expressions for the optimal state and control trajectories and of the optimal cost. These expressions enable the structure of the optimal solution to be analyzed in terms of the parameters of the problem. Moreover, it allows to obtain the solution of more general control problems, where the dependence of the optimal solution on the parameters of the problem has to be analyzed in view, for example, of a second optimization with respect to such parameters: an example of this form is discussed in Section 3.1.

- From a computational viewpoint, the integration of the Riccati differential equation, which leads to a heavy computational burden, is avoided. Instead, this method relies only on algebraic procedures that can be implemented by numerically robust routines.

- As a byproduct of the closed-form solution of our problem, we obtain a new result on the differential Riccati equation. More precisely, in Section 5 a new closed-form expression is established under extremely weak assumption for the solution of the matrix differential Riccati equation with assigned terminal condition.

- As described in Section 6.1, this method turns out to be a practical tool for a straightforward solution to the $\mathrm{H}_{2}$ decoupling with preview, where an input signal which is known in advance of a fixed time interval has to be decoupled from the output of an Linear Time Invariant (LIT) system. This problem has been addressed and solved in the literature, [28,15], by resorting to other approaches. For the same problem in the $\mathcal{H}_{\infty}$ setting we refer to $[11,5,8,4,9,27,10]$.
The solution presented in Section 6.1 has the advantage of providing in a very natural and simple way a closed-form expression of the decoupling filter.

Notation. Throughout this paper, the symbol $i \mathbb{R}$ denotes the imaginary axis in the complex plane. The image and the null-space of matrix $A$ are denoted by $\operatorname{im} A$ and $\operatorname{ker} A$, while $A^{\top}$ and $A^{+}$denote the transpose and the Moore-Penrose pseudo-inverse of $A$, respectively. The spectrum of $A$ is denoted by the symbol $\sigma(A)$.

\section{Statement of the Problem}

Consider the LTI state differential equation

$$
\dot{x}(t)=A x(t)+B u(t)
$$

where, for all $t \geq 0, x(t) \in \mathbb{R}^{n}$ is the state, $u(t) \in \mathbb{R}^{m}$ is the control input, $A \in \mathbb{R}^{n \times n}, B \in \mathbb{R}^{n \times m}$. Let $W \in \mathbb{R}^{s \times 2 n}$ be full-row rank and let $w \in \mathbb{R}^{s}$; consider the constraint

$$
W\left[\begin{array}{l}
x(0) \\
x(T)
\end{array}\right]=w
$$

Let

$$
\Pi:=\left[\begin{array}{cc}
Q & S \\
S^{\top} & R
\end{array}\right]=\Pi^{\top} \geq 0
$$

with $Q \in \mathbb{R}^{n \times n}, S \in \mathbb{R}^{n \times m}$ and $R \in \mathbb{R}^{m \times m}$. Assume $R>0$. Moreover, let

$$
\Theta:=\left[\begin{array}{ll}
\Theta_{1} & \Theta_{2} \\
\Theta_{2}^{\top} & \Theta_{3}
\end{array}\right]=\Theta^{\top} \geq 0
$$

with $\Theta_{1}, \Theta_{3}, \Theta_{3} \in \mathbb{R}^{n \times n}$. Finally, let $T>0$ be the length of the time horizon, and consider the quadratic performance index

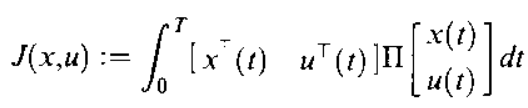

$$
\begin{aligned}
& +\left[\begin{array}{ll}
x^{\top}(0)-z_{0}^{\top} & x^{\top}(T)-z_{T}^{\top}
\end{array}\right] \Theta\left[\begin{array}{l}
x(0)-z_{0} \\
x(T)-z_{T}
\end{array}\right],
\end{aligned}
$$

where $z_{0}, z_{r} \in \mathbb{R}^{n}$. The optimal control problem dealt with is the following:

Problem 1: Find a measurable $u(t), t \in[0, T)$, and an absolutely continuous $x(t), t \in[0, T)$, minimizing the quadratic index $J(x, u)$ under the constraints (1) and (2).

Remark 1: The formulation of Problem 1 is very general, since it involves affine constraints on the extreme 
states (2) and since the differences between the extreme states and the target states $z_{0}$ and $z_{T}$ are quadratically penalized in $J(x, u)$. In particular:

1. By setting $W=\left[\begin{array}{ll}I_{n} & 0\end{array}\right], z_{T}=0, \Theta_{1}=\Theta_{2}=0$, Problem 1 reduces to the standard LQ problem, in which the initial state is assigned, $x(0)=w$, and the terminal state is weighted quadratically in the performance index through the penalty matrix $\Theta_{3}$;

2. By setting $W=I_{2 n}$ and $\Theta=0$, we obtain the fixed end-point $L Q$, in which the extreme states are both sharply assigned, $\left[\begin{array}{c}x(0) \\ x(T)\end{array}\right]=w$;

3. Problem 1 encompasses the case when the initial and terminal values $y(0)$ and $y(T)$ of an output $y(t)=C x(t)$ of the dynamical system described by (1) are constrained to be equal to $y_{0}$ and $y_{T}$, respectively, by taking $W=\operatorname{diag}(C, C)$, and $w=\left[\begin{array}{l}y_{0} \\ y_{T}\end{array}\right]$. This case is often referred to as the point-to-point $\mathrm{LQ}$, see [23] and references therein.

4. Many nonstandard LQ problems which can be useful in practice are particular cases of Problem 1. Consider for example an LQ problem in which the extreme states $x(0)$ and $x(\mathrm{~T})$ are not assigned, but they are constrained to be equal, that is, $x(0)=$ $x(T)$. Clearly, this case can be obtained by Problem 1 with $W=\left[\begin{array}{ll}I_{n} & -I_{n}\end{array}\right]$ and $w=0$. Alternatively, consider the case when the difference $x(0)-x(T)$ is not assigned but has to be quadratically penalized in the performance index with a matrix $\Delta=\Delta^{\top} \geq 0$ :

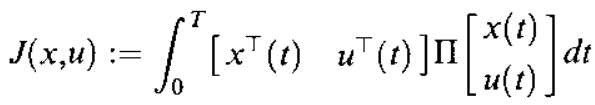

$$
\begin{aligned}
& +(x(0)-x(T))^{\top} \Delta(x(0)-x(T)) \text {. }
\end{aligned}
$$

A straightforward computation shows that this performance index can be brought back to the form given in (4) with

$$
\Theta=\left[\begin{array}{cc}
\Delta & -\Delta \\
-\Delta & \Delta
\end{array}\right]
$$

and $z_{0}=z_{T}=0$.

The set of Pontryagin-type equations corresponding to Problem 1 are collected in the following lemma. As is well-known, in general the Pontryagin equations give only necessary conditions for optimality. In the case considered, however, since the underlying system, the performance index and the extreme constraints are convex functions in their arguments, the Pontryagin conditions are also sufficient for an optimum, as the following lemma shows.

Lemma 1: If $u(t)$ and $x(t)$ are optimal for Problem 1, then $\lambda(t) \in \mathbb{R}^{n}, t \in[0, T]$ and $\nu \in \mathbb{R}^{s}$ exist such that $x(t), \lambda(t), u(t)$ and $\nu$ satisfy for all $t \in[0, T)$ the following set of equations:

$$
\begin{aligned}
& \dot{x}(t)=A x(t)+B u(t), \\
& W\left[\begin{array}{c}
x(0) \\
x(T)
\end{array}\right]-w=0, \\
& \dot{\lambda}(t)=-Q x(t)-S u(t)-A^{\top} \lambda(t), \\
& {\left[\begin{array}{c}
-\lambda(0) \\
\lambda(T)
\end{array}\right]=\Theta\left[\begin{array}{c}
x(0)-z_{0} \\
x(T)-z_{T}
\end{array}\right]+W^{\top} \nu,} \\
& R u(t)+S^{\top} x(t)+B^{\top} \lambda(t)=0 .
\end{aligned}
$$

Conversely, if equations (5)-(9) admit solutions $x(t)$, $u(t), \lambda(t), \nu$, then $x(t), u(t)$ minimize $J(x, u)$ subject to the constraints $(1)-(2)$.

A complete proof of this generalized version of the Pontryagin equations - where differently from the standard case the constraints on the initial and terminal states are coupled - can be found in $[22$, p. 6], where sufficiency is proved along the same lines of Theorem 1 in [19]. A detailed discussion on the existence and uniqueness of solutions of Problem 1, and hence of equations (5)-(9), will be carried out in Section 4.

Since $R$ is assumed to be invertible, equation (9) can be solved in $u(t)$ yiclding

$$
u(t)=-R^{-1}\left(S^{\top} x(t)+B^{\top} \lambda(t)\right)
$$

The latter can be substituted in (5) and (7), so as to obtain the so-called Hamiltonian differential equation

$$
\dot{p}(t)=H p(t), \quad t \in[0, T),
$$

where $p(t):=\left[\begin{array}{ll}x^{\top}(t) & \lambda^{\top}(t)\end{array}\right]^{\top}$ and the Hamiltonian matrix $H$ is defined as

$$
H:=\left[\begin{array}{cc}
A-B R^{-1} S^{\top} & -B R^{-1} B^{\top} \\
-Q+S R^{-1} S^{\top} & -A^{\top}+S R^{-1} B^{\top}
\end{array}\right],
$$

see for example, [1, Chapter 2]. Hence, equations (10), (11), (6), (8) are necessary and sufficient for an optimum of Problem 1, as well, since they are obtained by linearly combining (5-9). 


\section{Hamiltonian Trajectories and Optimal Control}

Due to the linearity of the Hamiltonian differential equation (11), a straightforward parameterization of the solutions of (11) is given by

$$
p(t)=e^{H t} p_{0}, \quad t \in[0, T],
$$

where the constant $p_{0}$ can be found by imposing the boundary conditions on (13). However, in this paper we are interested in a more explicit representation of the solutions of the Hamiltonian differential equation (11), see Remark 2. To this end, let us assume that:

(A1) the pair $(A, B)$ is sign-controllable;

(A2) $H$ has no eigenvalues on $i \mathbb{R}$.

We recall that the sign-controllability of the pair $(A, B)$ means that the set of uncontrollable eigenvalues of $A$ does not contain pairs of elements in the form $(\lambda,-\bar{\lambda}),[26,16]$. Assumption (A1) is the weakest form of controllability: It is weaker than the assumption of reachability, and even than that of stabilizability of the pair $(A, B)$. Indeed, it generically holds even in the extreme case when $B=0$.

Now, consider the ARE

$$
P A+A^{\top} P-(S+P B) R^{-1}\left(S^{\top}+B^{\top} P\right)+Q=0 .
$$

To any solution $P=P^{\top} \in \mathbb{R}^{n \times n}$ of (14) corresponds the closed-loop matrix

$$
A_{P}:=A-B K_{P}, \quad K_{P}:=R^{-1}\left(S^{\top}+B^{\top} P\right) .
$$

Recall that a symmetric solution $P_{u}$ of (14) is said to be unmixed if, by defining $A_{u}:=A_{P_{u}}$ and $K_{u}:=K_{P_{u}}$, $\lambda,-\bar{\lambda} \in \sigma\left(A_{u}\right)$ implies $\Re e(\lambda)=0,[26,25]$. However, in this paper we are interested in a more stringent property: we define strongly unmixed a solution $P_{u}=P_{u}^{\top}$ of (14) such that the spectrum of the corresponding $A_{u}$ does not contain mirrored pairs, that is, $\lambda \in \sigma\left(A_{u}\right)$ implies $-\bar{\lambda} \notin \sigma\left(A_{u}\right)$. Since $A_{u}$ is real, this is equivalent to the fact that $\sigma\left(A_{u}\right)$ does not contain opposite pairs $(\lambda,-\lambda)$. Clearly, if $P_{u}$ is unmixed and none of the eigenvalues of $A_{u}$ lay on the imaginary axis, then $P_{u}$ is also strongly unmixed. Note also that given a solution $P=P^{\top}$ of (14) and the corresponding closed-loop matrix $A_{P}$ defined in (15), the Lyapunov equation

$$
A_{P} Y+Y A_{P}^{\top}+B R^{-1} B^{\top}=0
$$

has a unique solution $Y=Y^{\top} \in \mathbb{R}^{n \times n}$ if and only if $P$ is strongly unmixed, [17, Theorem 5.2.2].
Lemma 2: Let assumptions (A1)-(A2) hold. Then, the $A R E$ (14) admits a strongly unmixed solution.

Proof: In [25, Theorem 5] it is shown that the signcontrollability of $(A, B)$ ensures the existence of an unmixed solution $P_{u}$ of the ARE (14). Moreover, by virtue of assumption (A2), $P_{u}$ is also strongly unmixed, since $\sigma\left(A_{u}\right) \subset \sigma(H)$, [21, Theorem 6]

Now we are ready to present the main result of the paper, consisting of an explicit formula parameterizing all the solutions of the Hamiltonian differential equation (11).

Theorem 1: Let assumptions $(A 1)-(A 2)$ hold. Let $P_{u}$ be a strongly unmixed solution of (14), let $A_{u}$ be the corresponding closed-loop system matrix and $Y$ be the corresponding solution of (16). The set of solutions of (11) is parameterized in $p, q \in \mathbb{R}^{n}$ as

$$
p(t)=J_{1} e^{A_{u} t} p+J_{2} e^{A_{\mu}^{\top}(T-t)} q, \quad t \in[0, T],
$$

where $J_{1}:=\left[\begin{array}{c}I_{n} \\ P_{u}\end{array}\right]$ and $J_{2}:=\left[\begin{array}{c}Y \\ P_{u} Y-I_{n}\end{array}\right]$.

Proof: We first prove that (17) satisfies (11). By exploiting (14), (15) and (16), we easily get $H J_{1}=J_{1} A_{t}$ and $H J_{2}=-J_{2} A_{u}^{\top}$. By using these equalities, a direct substitution shows that (17) satisfies (11). Conversely, we will prove that all the solutions of (11) can be expressed by means of (17) for suitable values of $p$ and $q$. First, note that the order of the linear differential equation (11) is $2 n$, hence it admits $2 n$ linearly independent solutions. We will show that we may select $2 n$ linearly independent solutions from (17). To this aim, we first need to show that $U(t):=$ $\left[\begin{array}{ll}J_{1} e^{A_{u} t} & J_{2} e^{A_{u}^{\top}(T-t)}\end{array}\right]$ is nonsingular for some $t \in[0, T]$. This condition is easily checked. In fact, since $H J_{1}=J_{1} A_{u}$ and $H J_{2}=-J_{2} A_{u}^{\top}$ as already observed, the subspaces $\mathcal{J}_{1}:=\operatorname{im} J_{1}$ and $\mathcal{J}_{2}:=\operatorname{im} J_{2}$ are $H$ invariant and the eigenvalues of $H$ restricted to $\mathcal{J}_{1}$ and to $\mathcal{J}_{2}$ are the eigenvalues of $A_{u}$ and of $-A_{u}^{\top}$, respectively. Note that $A_{u}$ and $-A_{u}^{\top}$ have no eigenvalues in common since $P_{u}$ is strongly unmixed. Hence, $\mathcal{J}_{1} \cap \mathcal{J}_{2}=\{0\}$. Finally, since $e^{A_{u} t}$ and $e^{A_{u}^{\top}(T-t)}$ are full rank for all $t \in[0, T], U(t)$ is nonsingular for all $t \in[0, T]$. Now, take $2 n$ linearly independent vectors $\pi_{i}:=\left[\begin{array}{c}p_{i} \\ q_{i}\end{array}\right] \in \mathbb{R}^{2 n}, i=1, \ldots, 2 n$, and define the corresponding $2 n$ trajectories $p_{i}(t)$ of (17) using $p_{i}, q_{i}$. We get $p_{i}(t)=U(t) \pi_{i}$ for all $t \in[0, T]$. Hence, $\sum_{i=1}^{2 n} k_{i} p_{i}(t)=0$ for all $t \in[0, T]$ implies that $\sum_{i=1}^{2 n} k_{i} \pi_{i}=0$, since $U(t)$ is nonsingular for some $t \in[0, T]$. It follows that $k_{i}=0$ for all $i=1, \ldots, 2 n$, since $\left(\pi_{i}\right)_{i=1, \ldots, 2 n}$ are linearly 
independent. We may conclude that (17) gives rise to $2 n$ linearly independent trajectories.

The following result provides the complete solution of Problem 1.

Theorem 2: Let $V$ be a basis matrix ${ }^{l}$ of the null-space of $W$. Define $F:=e^{A_{u} T}$. Moreover, let $P:=\operatorname{diag}\left(-P_{u}, P_{u}\right)$ and $\quad z:=\left[\begin{array}{l}z_{0} \\ z_{T}\end{array}\right] . \quad$ Define $L:=\left[\begin{array}{cc}I_{n} & Y F^{\top} \\ F & Y\end{array}\right]$, $U:=\left[\begin{array}{cc}0 & -F^{\top} \\ 0 & I_{n}\end{array}\right]$

and

$$
N:=\left[\begin{array}{c}
W L \\
V^{\top}[(P-\Theta) L-U]
\end{array}\right], \quad \xi:=\left[\begin{array}{c}
w \\
-V^{\top} \Theta z
\end{array}\right] .
$$

Problem 1 admits solutions if and only if $\xi \in \mathrm{im} N$.

If this is the case, let $K_{N}$ be a basis matrix of the nullspace of $N$, and define

$$
\mathcal{P}:=\left\{\pi=N^{+} \xi+K_{N} v: v \text { arbitrary }\right\} .
$$

Then, the set of optimal solutions of Problem $I$ is parameterized by

$$
\left[\begin{array}{l}
x(t) \\
u(t)
\end{array}\right]=\left[\begin{array}{cc}
e^{A_{u} t} & Y e^{A_{u}^{\top}(T-t)} \\
-K_{u} e^{A_{u} t} & -R^{-1}\left(S^{\top} Y+B^{\top} P_{u} Y-B^{\top}\right) e^{A_{u}^{\top}(T-t)}
\end{array}\right] \pi, \quad \pi \in P
$$

enable $x(t), \lambda(t)$ and $u(t)$ to be determined. Then $\nu$ can be computed from (8), since $W$ is full-row rank. As a result, a solution of (5)-(9) exists: such a solution is optimal for Problem 1 by virtue of Lemma 1. Finally, (19) parameterizes the set of solutions of the linear equation $N \pi=\xi$ in the case when $\xi \in \operatorname{im} N$. For all $\pi \in P$, the optimal state and control functions satisfy (17) and (10), yielding (20) after straightforward algebraic manipulations.

Remark 2: In Theorem 2 we have shown how the parameterization (17) of the solutions of the Hamiltonian differential equation (11) given in Theorem 1 can be employed in order to provide a complete solution to Problem 1. As aforementioned, an alternative way of solving Problem 1 consists of using the formula (13) as follows. Let $e^{H T}=\left[\begin{array}{ll}\Psi_{11} & \Psi_{12} \\ \Psi_{21} & \Psi_{22}\end{array}\right]$ be partitioned comformably with $\left[\begin{array}{l}x(T) \\ \lambda(T)\end{array}\right] ; \quad$ on noting that $\quad p(t)=e^{H t} p_{0}$, where
Proof: Consider a solution of (5)-(9). By virtue of Theorem 1, the fact that this solution satisfies (5), (7) and (9) implies that its structure is that given by (17) for suitable values of $p$ and $q$. Moreover, this solution satisfies the boundary conditions (6), and (8). By imposing such equations on (17) evaluated at $t=0$ and $t=T$, we find the equalities

$$
\begin{aligned}
& W\left[\begin{array}{c}
p+Y F^{\top} q \\
F p+Y q
\end{array}\right]=w, \\
& {\left[\begin{array}{c}
-P_{u} p-\left(P_{u} Y-I_{n}\right) F^{\top} q \\
P_{u} F p+\left(P_{u} Y-I_{n}\right) q
\end{array}\right]=\Theta\left[\begin{array}{r}
p+Y F^{\top} q-z_{0} \\
F p+Y q-z_{T}
\end{array}\right]} \\
& +W^{\top} \nu .
\end{aligned}
$$

By premultiplying the second equation by $V^{\top}$, we obtain the compact equation $N \pi=\xi$ in the unknown $\pi:=\left[\begin{array}{l}p \\ q\end{array}\right]$.

Hence, we have shown that $N \pi=\xi$ follows from (5) to (9). But the converse is true as well: if $\xi \in \operatorname{im} N$ and $\pi:=\left[\begin{array}{c}p \\ q\end{array}\right]$ satisfies $N \pi=\xi$, equations (17) and (10) ${ }^{\text {II }}$ the case when $\operatorname{ker} W=\{0\}$ we consider $V$ to be void. $p_{0}:=\left[\begin{array}{l}x(0) \\ \lambda(0)\end{array}\right]$, we can eliminate $x(T)=\Psi_{11} x(0)+$ $\Psi_{12} \lambda(0)$ and $\lambda(T)=\Psi_{21} x(0)+\Psi_{22} \lambda(0)$ from (6) and (8), so as to obtain the equations

$$
\begin{aligned}
& W\left[\begin{array}{l}
x(0) \\
x(T)
\end{array}\right]-w=0, \\
& {\left[\begin{array}{c}
-\lambda(0) \\
\lambda(T)
\end{array}\right]=\Theta\left[\begin{array}{c}
x(0)-z_{0} \\
x(T)-z_{T}
\end{array}\right]+W^{\top} \nu,}
\end{aligned}
$$

along the same lines of the proof of Theorem 2. It is easy to write these equations in the compact form $N^{\prime} p_{0}=\xi$, where now $p_{0}$ can be regarded as the parameter to be replaced in $p(t)=e^{H t} p_{0}$ to find the optimal state and costate trajectories. This solution is more general than the one shown based on (17) in Theorem 1 , since no assumptions are necessary on the pair $(A, B)$ and on the eigenvalues of the Hamiltonian matrix. Nevertheless, the expression used for the determination of the parameter $p_{0}$ depends on the matrices $\Psi_{11}$, $\Psi_{12}, \Psi_{21}, \Psi_{22}$; an explicit relation between these submatrices of $e^{H T}$ and the problem parameters is in general not available. As such, if we use the trivial parameterization (13) it remains unclear how to 
express the optimal solution in terms of the variations of the problem data. On the contrary, this kind of investigation is possible in the case we use the parameterization (17) in view of the many results available in the literature estabilishing how the solutions of AREs vary in terms of the variation of the matrices $A$, $B$ and $\Pi,[17,24]$.

Moreover, in the case where the pair $(A, B)$ is stabilizable, a further and more relevant advantage connected with the use of (17) over (13) for the solution of Problem 1 is numerical, as discussed in Section 6.

In the following theorem, a simple formula for the computation of the optimal cost as a quadratic form of the problem data is presented.

Theorem 3: Consider the matrices $P, L, U, z, N$ and $\xi$ defined in Theorem 2 , and let $X:=\operatorname{diag}(-Y, Y)$. Define

$$
\Upsilon:=\left[\begin{array}{cc}
N^{+\top}\left[L^{\top}(\Theta-P) L+U^{\top} X U\right] N^{+} & -N^{+\top} L^{\top} \Theta \\
-\Theta^{\top} L N^{+} & \Theta
\end{array}\right]
$$

and

$$
G:=\left[\begin{array}{cc}
I_{s} & 0 \\
0 & -V^{\top} \Theta \\
0 & I_{2 n}
\end{array}\right]
$$

If Problem 1 has solutions, the optimal value $J^{*}$ of the functional $J(x, u)$ is given by the quadratic form

$$
J^{*}=\left[\begin{array}{ll}
w^{\top} & z^{\top}
\end{array}\right] G^{\top} \Upsilon G\left[\begin{array}{c}
w \\
z
\end{array}\right]
$$

Proof: From (10), the value $c(x, u)$ of the integral in $J(x, u)$ associated with the optimal solution $x(t), u(t)$ of Problem 1 can be written as

$$
c(x, u)=\int_{0}^{T} p^{\top}(t) \Gamma p(t) d t
$$

with $\quad \Gamma:=\operatorname{diag}\left(Q-S R^{-1} S^{\top}, B R^{-1} B^{\top}\right), \quad p(t):=$ $\left[\begin{array}{l}x(t) \\ \lambda(t)\end{array}\right]$. Let now be $\bar{J}:=\left[\begin{array}{cc}0 & I_{n} \\ I_{n} & 0\end{array}\right]$. A simple computation yields $H^{\top} \bar{J}+\bar{J} H=-2 \Gamma$, that can be used in (24). By (11)

$$
\begin{aligned}
c(x, u) & =-\frac{1}{2} \int_{0}^{T} \frac{d}{d t}\left(p^{\top}(t) p(t)\right) d t \\
& =x^{\top}(0) \lambda(0)-x^{\top}(T) \lambda(T) .
\end{aligned}
$$

By using this formula in $J(x, u)$, we obtain an expression of $J^{*}$ in terms of $x(0), \lambda(0), x(T)$ and $\lambda(T)$.
Since the optimal state and costate satisfy (17), an expression of $J^{*}$ as a quadratic form of $z$ and of the value $\pi=\left[\begin{array}{l}p \\ q\end{array}\right]$ corresponding to the optimal solution can be determined after simple algebraic manipulations:

$$
\begin{aligned}
J^{*}= & {\left[\begin{array}{ll}
\pi^{\top} & z^{\top}
\end{array}\right] } \\
& \times\left[\begin{array}{cc}
L^{\top}(\Theta-P) L+U^{\top} X U & -L^{\top} \Theta \\
-\Theta^{\top} L & \Theta
\end{array}\right]\left[\begin{array}{l}
\pi \\
z
\end{array}\right] .
\end{aligned}
$$

Finally, consider (19), and note that different values of $v$ do not affect the corresponding value of the cost. Hence, we may choose $v=0$, and an expression of $J^{*}$ as a quadratic form in $\xi$ and $z$ is easily derived. Now, since

$$
\left[\begin{array}{l}
\xi \\
z
\end{array}\right]=\left[\begin{array}{c}
w \\
-V^{\mathrm{T}} \Theta z \\
z
\end{array}\right]=G\left[\begin{array}{l}
w \\
z
\end{array}\right]
$$

the expression (23) follows straightforwardly.

Remark 3: In the case when the pair $(A, B)$ is not signcontrollable, but the non sign-controllable part is nonobservable in the performance index, a parameterization of the solutions of the Hamiltonian differential equation can still be established. In order to clarify the meaning of sign-controllable and non signcontrollable part of the state, we first derive a signcontrollability form of the pair $(A, B)$. Consider the pair $(A, B)$ in the reachability canonical form

$$
A=\left[\begin{array}{cc}
A_{11} & 0 \\
A_{21} & A_{22}
\end{array}\right] \quad \text { and } \quad B=\left[\begin{array}{c}
0 \\
B_{2}
\end{array}\right]
$$

where the pair $\left(A_{22}, B_{2}\right)$ is reachable. Define $\Lambda:=$ $\sigma\left(A_{11}\right) \cap \sigma\left(-A_{11}\right)$. Hence, there exists $T$ such that $T^{-1} A_{11} T=\operatorname{diag}\left(A_{11}^{\prime}, A_{11}^{\prime \prime}\right)$ with $\sigma\left(A_{11}^{\prime}\right)=\Lambda$. Hence, by performing a change of coordinates in the original system by means of the matrix $U:=\operatorname{diag}(T, I)$, we obtain

$$
\begin{aligned}
U^{-1} A U & =\left[\begin{array}{cc}
T^{-1} A_{11} T & 0 \\
A_{21} T+A_{22} & A_{22}
\end{array}\right] \\
& =\left[\begin{array}{ccc}
A_{11}^{\prime} & 0 & 0 \\
0 & A_{11}^{\prime \prime} & 0 \\
A_{21}^{\prime} & A_{21}^{\prime \prime} & A_{22}
\end{array}\right], \quad U^{-1} B=\left[\begin{array}{c}
0 \\
0 \\
B_{2}
\end{array}\right],
\end{aligned}
$$


where we have partitioned $A_{21} T+A_{22}$ as $\left[\begin{array}{ll}A_{21}^{\prime} & A_{21}^{\prime \prime}\end{array}\right]$. The pair

$$
\left[\begin{array}{cc}
A_{11}^{\prime \prime} & 0 \\
A_{21}^{\prime \prime} & A_{22}
\end{array}\right], \quad\left[\begin{array}{c}
0 \\
B_{2}
\end{array}\right]
$$

is sign-controllable: in fact, the pair $\left(A_{22}, B_{2}\right)$, as already observed, is reachable, while the submatrix $A_{i 1}^{\prime \prime}$ has not mirrored pairs of eigenvalues with respect to the imaginary axis (and has no eigenvalues on the imaginary axis).

Let now the pair $(A, B)$ be in the sign-controllability form, that is,

$$
A=\left[\begin{array}{cc}
A_{1} & 0 \\
A_{3} & A_{2}
\end{array}\right] \text { and } B=\left[\begin{array}{c}
0 \\
B_{2}
\end{array}\right]
$$

where the pair $\left(A_{2}, B_{2}\right)$ is sign-controllable and $\sigma\left(A_{1}\right)=\sigma\left(-A_{1}\right)$. Let the non sign-controllable part of the state be non observable in the performance index, so that the matrices $Q$ and $S$ are partitioned accordingly to this basis as

$$
Q=\left[\begin{array}{cc}
0 & 0 \\
0 & Q_{2}
\end{array}\right] \text { and } S=\left[\begin{array}{c}
0 \\
S_{2}
\end{array}\right]
$$

and $A_{3}=0$. If the Hamiltonian matrix referred to the sole sign-controllable part, which can be written as

$$
H_{2}:=\left[\begin{array}{cc}
A_{2}-B_{2} R^{-1} S_{2}^{\top} & -B_{2} R^{-1} B_{2}^{\top} \\
-Q_{2}+S_{2} R^{-1} S_{2}^{\top} & -A_{2}^{\top}+S_{2} R^{-1} B_{2}^{\top}
\end{array}\right],
$$

has no eigenvalues on the imaginary axis, a matrix $P_{2}$ and a matrix $Y_{2}$ exist satisfying the ARE referred to the sole sign-controllable part and the corresponding closed-loop Lyapunov equation, respectively, so that the matrices

$$
P=\left[\begin{array}{cc}
0 & 0 \\
0 & P_{2}
\end{array}\right] \text { and } Y=\left[\begin{array}{cc}
0 & 0 \\
0 & Y_{2}
\end{array}\right]
$$

are the solutions of the ARE and of the corresponding closed-loop Lyapunov equation referred to the complete system, respectively. By using these matrices in (17), we obtain a parameterization of the set of solutions of the Hamiltonian differential equation over the time interval $[0, T)$.

\subsection{Examples}

Consider the pair $(A, B)$ given by

$$
A=\left[\begin{array}{ccc}
-3 & 0 & 0 \\
0 & 2 & 0 \\
0 & -1 & 1
\end{array}\right], \quad B=\left[\begin{array}{l}
0 \\
0 \\
1
\end{array}\right]
$$

which is clearly sign-controllable (but not stabilizable nor antistabilizable), and a Popov matrix $\Pi$ in which

$$
Q=\frac{1}{2}\left[\begin{array}{ccc}
15 & 3 & 3 \\
3 & 12 & 3 \\
3 & 3 & 15
\end{array}\right], \quad S=\left[\begin{array}{c}
0 \\
-1 \\
0
\end{array}\right], \quad R=\frac{1}{2}
$$

so that $\Pi=\Pi^{\top} \geq 0$. Let $z_{0}=\left[\begin{array}{lll}1 & 2 & 3\end{array}\right]^{\top}$ and $z_{T}=$ $\left[\begin{array}{lll}0 & 1 & 0\end{array}\right]^{\top}$. Let $\Theta$ be partitioned as

$$
\Theta:=\left[\begin{array}{cc}
\Theta_{1} & \Theta_{2} \\
\Theta_{2}^{\top} & \Theta_{3}
\end{array}\right]
$$

where

$$
\begin{array}{ll}
\Theta_{1}=\left[\begin{array}{ccc}
12 & 0 & 0 \\
0 & 0 & 0 \\
0 & 0 & 0
\end{array}\right], & \Theta_{2}=\left[\begin{array}{lll}
0 & 2 & 0 \\
0 & 0 & 0 \\
0 & 0 & 0
\end{array}\right], \\
\Theta_{3}=\left[\begin{array}{llc}
0 & 0 & 0 \\
0 & 4 & 0 \\
0 & 0 & 24
\end{array}\right] . &
\end{array}
$$

Moreover, consider the following constraints on the extreme states, in which $T=2$ :

$$
\begin{aligned}
& x_{1}(0)-x_{3}(0)=2, \\
& x_{2}(0)+x_{3}(T)=2, \\
& x_{2}(T)=1 .
\end{aligned}
$$

It is easily seen that the matrices

$$
\begin{aligned}
& P_{u}=\frac{1}{2}\left[\begin{array}{ccc}
1 & 0 & -3 \\
0 & -2 & 0 \\
-3 & 0 & -3
\end{array}\right] \text { and } \\
& Y=\frac{1}{4}\left[\begin{array}{ccc}
0 & 0 & 0 \\
0 & 0 & 0 \\
0 & 0 & -1
\end{array}\right]
\end{aligned}
$$

are an unmixed solution of the ARE corresponding to the eigenvalues $\{-3,1,2\}$ of the Hamiltonian matrix, and the corresponding solution of the Lyapunov equation, respectively. First, we compute the parameters $p$ and $q$ by means of the formula $N \pi=\xi$ in Theorem 2. In this case $N$ is invertible, so that 
$\pi=N^{-1} \xi$. Straightforward computations yield the following expressions for $p$ and $q$ :

$$
\begin{aligned}
& {\left[\begin{array}{l}
p_{1} \\
p_{2} \\
p_{3}
\end{array}\right]=\frac{1}{d}\left[\begin{array}{c}
\bar{p}_{1} \\
d e^{-4} \\
\bar{p}_{3}
\end{array}\right] \simeq\left[\begin{array}{c}
1.0749 \\
0.0183 \\
-0.4690
\end{array}\right] \text { and }} \\
& {\left[\begin{array}{l}
q_{1} \\
q_{2} \\
q_{3}
\end{array}\right]=\frac{1}{d}\left[\begin{array}{l}
\bar{q}_{1} \\
\bar{q}_{2} \\
\bar{q}_{3}
\end{array}\right] \simeq\left[\begin{array}{c}
-2.9712 \\
-0.9424 \\
0.0006
\end{array}\right] .}
\end{aligned}
$$

where

$$
\begin{aligned}
d:= & 121-784 e^{12}-480 e^{14}-49 e^{16}+1584 e^{28}, \\
\bar{p}_{1}:= & 7 e^{2}\left(3-18 e^{4}+12 e^{8}-84 e^{10}-48 e^{12}\right. \\
& -80 e^{14}+21 e^{16}+200 e^{18}-42 e^{20} \\
& \left.-40 e^{22}+244 e^{26}\right), \\
\bar{p}_{3}:= & -\frac{1}{2}\left(484-42 e^{2}-224 e^{4}+252 e^{6}+497 e^{8}\right. \\
& -168 e^{10}-2009 e^{12}-1752 e^{14}+2688 e^{16} \\
& +126 e^{18}-6720 e^{20}-252 e^{22}+1344 e^{24} \\
& \left.+1464 e^{28}\right), \\
\bar{q}_{1}:= & -2 e^{-4}\left(-96+213 e^{4}-21 e^{8}+147 e^{10}\right. \\
& +672 e^{12}+500 e^{14}-1176 e^{16}-1070 e^{18} \\
& \left.+70 e^{22}-427 e^{26}-1188 e^{28}+2376 e^{32}\right) \\
\bar{q}_{2}:= & e^{-8}\left(2485-4764 e^{4}-945 e^{8}-126 e^{10}\right. \\
& -14630 e^{12}-9576 e^{14}+28910 e^{16} \\
& +19776 e^{18}+10192 e^{20}+1680 e^{22} \\
& \left.-7616 e^{24}-3402 e^{26}+41048 e^{28}-82240 e^{32}\right), \\
\bar{q}_{3}:= & 14 e^{-4}\left(32-71 e^{4}+7 e^{8}+72 e^{10}-252 e^{12}\right. \\
& \left.-60 e^{14}+560 e^{16}+120 e^{18}-112 e^{20}+208 e^{24}\right) .
\end{aligned}
$$

Now, by using (20) the expression of the optimal state trajectory and control law can be determined in closed form:

$$
\begin{aligned}
x_{1}(t)= & \frac{1}{4}\left(e^{-t}+3 e^{3 t}\right) p_{1}+\frac{1}{4}\left(3 e^{3 t}-3 e^{-t}\right) p_{3} \\
& +\frac{1}{2} e^{t-2} q_{1}-\frac{1}{2} e^{t-2} q_{3},
\end{aligned}
$$

$$
\begin{aligned}
x_{2}(t)= & e^{2 t} p_{2}, \\
x_{3}(t)= & \frac{1}{4}\left(e^{3 t}-e^{-t}\right) p_{1}+\frac{1}{4}\left(3 e^{-t}+e^{3 t}\right) p_{3} \\
& -\frac{1}{2} e^{3(2-t)} q_{1}-\frac{1}{6} e^{3(2-t)} q_{3}, \\
u(t)= & \frac{1}{2}\left(3 e^{3 t}-e^{-t}\right) p_{1}+\frac{3}{2}\left(e^{-t}+e^{3 t}\right) p_{3} .
\end{aligned}
$$

Finally, the optimal cost is computed in closedform as

$$
J^{*}=\frac{c}{d} \simeq 104.3325,
$$

where

$$
\begin{aligned}
c:= & 2485 e^{-8}-9976 e^{-4}+9878-252 e^{2} \\
& -13286 e^{4}-8568 e^{6}+58842 e^{8}+40392 e^{10} \\
& -52920 e^{12}-39048 e^{14}-20671 e^{16}-6804 e^{18} \\
& +54152 e^{20}+10248 e^{22}-164480 e^{24} \\
& +168232 e^{28} .
\end{aligned}
$$

In Fig. 1, the optimal state and input functions are presented. To the best of our knowledge, this problem has not been solved (and is not easy to solve) with the classical approach to the LQ problem.

Remark 4: The solution of Problem 1 and the optimal cost have been explicitly written as parametric functions of the vector $\xi$. This form of the optimal solution is particularly convenient to deal with parametric problems, in which the dependence of the optimal

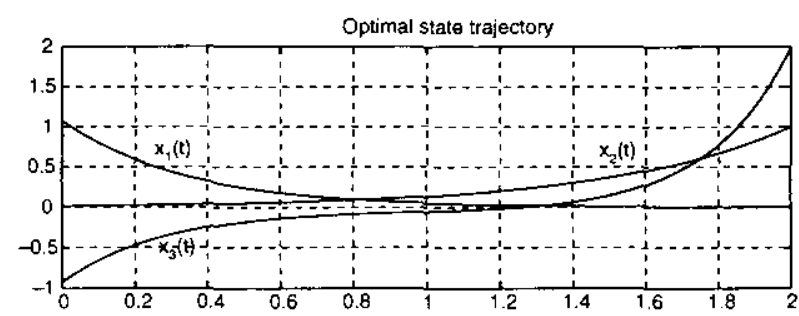

Optimal control

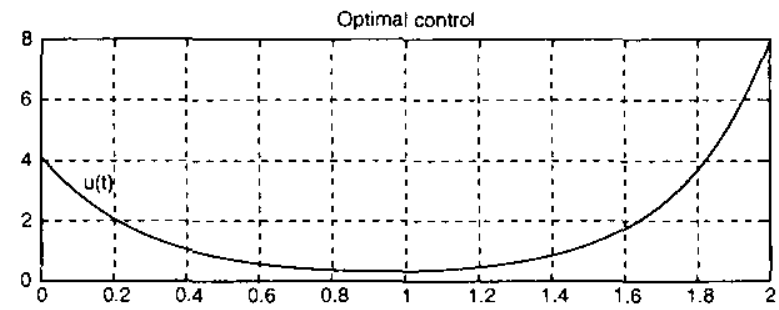

Fig. 1. Optimal state trajectory and control function. 
state and input trajectories and of the optimal cost has to be analyzed as a function of the parameters of the problem. As an example, consider the problem described in Example 1, where however $z_{0}$ may vary around its nominal value $\bar{z}_{0}=\left[\begin{array}{lll}1 & 2 & 3\end{array}\right]^{\top}$. In other words, $z_{0}=\bar{z}_{0}+\Delta z_{0}$. We are interested in analyzing the variations of the optimal cost $J^{*}$ with respect to small variations $\Delta z_{0}$. This corresponds to expressing $J^{*}$ as a function of $z_{0}$ and to computing the gradient $\left.\frac{\partial f}{\partial z_{0}}\right|_{z_{0}=\bar{z}_{0}}$. In the present case it can be easily checked that

$$
\left.\frac{\partial J^{*}}{\partial z_{0}}\right|_{z_{0}=\bar{z}_{0}} \simeq\left[\begin{array}{c}
-1.7972 \\
0 \\
0
\end{array}\right]
$$

Similarly, if some of the parameters of the problem are not assigned, a further optimization can be achieved with respect to these parameters. For example, consider the problem of Example 1 where, however, the target states $z_{0}$ and $z_{T}$ are not assigned, but they are constrained to be equal, that is, $z_{0}=z_{T}$; since the minimum $J^{*}$ of the performance index $J(x, u)$ can be expressed as a quadratric form of $w, z_{0}$ and $z_{T}$, by taking the constraint $z_{0}=z_{T}$ into account, this minimum can be also expressed as a quadratic form $q\left(w, z_{0}\right)$ of $w, z_{0}$. The minimum of this problem can be obtained by performing a minimization of the quadratic form $q\left(w, z_{0}\right)$ over $z_{0}$, which is standard and easy to solve.

\section{Existence and Uniqueness of an Optimum}

In Theorem 2 it has been shown that a necessary and sufficient condition for optimality is that the vector $\xi$ lies in the range of the matrix $N$, which is equivalent to the fact that equations (21)-(22) admit solutions for some $p$ and $q$. Now, we are interested in showing that the existence of a state trajectory and a control function satisfying the constraints (1) and (2) implies the existence of a solution for Problem 1. This condition is more informative than that represented by the inclu$\operatorname{sion} \xi \in \operatorname{im} N$, since it is equivalent to the fact that the first block-row $w$ of $\xi$ lies in the range of the first block-row $W L$ of $N$, that is, $w \in \operatorname{im} W L$.

The following theorem addresses this issue.

Theorem 4: Problem 1 admits solutions if and only if a trajectory of (1) exists satisfying the constraint (2).

Proof: First, note that the only if part of the proof is obvious, since the solution of Problem 1 is indeed a trajectory of (1) satisfying the constraint (2). Now, we assume that (1) and (2) admit solutions. Denote by $\left[\begin{array}{l}x_{0} \\ x_{T}\end{array}\right]$ any vector of $\mathbb{R}^{2 n}$ such that $W\left[\begin{array}{l}x_{0} \\ x_{T}\end{array}\right]=w$. The set of initial and terminal states satisfying the terminal constraint (6) can be parameterized as

$$
\left[\begin{array}{l}
x(0) \\
x(T)
\end{array}\right]=V \sigma+\left[\begin{array}{l}
x_{0} \\
x_{T}
\end{array}\right]
$$

where $V$ is a basis matrix of the null-space of $W$ and $\sigma$ is an arbitrary vector. Moreover, (1) represents a constraint on the extreme states $x(0)$ and $x(T)$ that can be written as

$$
x(T)=e^{A T} x(0)+R_{0} \tau,
$$

where $R_{0}$ is a basis matrix of the reachable subspace from the origin, that is, $\operatorname{im} R_{0}=$ $\operatorname{im}\left[\begin{array}{llll}B & A B & \ldots & A^{n-1} B\end{array}\right]$, and $\tau$ is an arbitrary vector. From (26) and (27) it follows that a trajectory of system (1) exists satisfying (2), if and only if the initial state $x(0)$ satisfies

$$
A_{0} x(0)=V \sigma-\bar{R} \tau+\bar{x}
$$

for some vectors $\sigma$ and $\tau$, where $\bar{R}:=\left[\begin{array}{c}0 \\ R_{0}\end{array}\right]$, $A_{0}:=\left[\begin{array}{c}I_{n} \\ e^{A T}\end{array}\right], \quad \bar{x}:=\left[\begin{array}{c}x_{0} \\ x_{T}\end{array}\right] . \quad$ Now define $A_{1}:=$ $\left[\begin{array}{ll}V & -\bar{R}\end{array}\right], \bar{A}:=\left[\begin{array}{ll}A_{0} & A_{1}\end{array}\right]$ and $\rho:=\left[\begin{array}{l}\sigma \\ \tau\end{array}\right]$. Hence, equation (28) can be written as

$$
\bar{A}\left[\begin{array}{c}
x(0) \\
\rho
\end{array}\right]=\bar{x}
$$

Hence, the set of initial states for which an input trajectory exists such that the corresponding state evolution satisfies $W\left[\begin{array}{l}x(0) \\ x(T)\end{array}\right]=w$ may be parameterized as

$$
x(0)=\left[\begin{array}{ll}
I_{n} & 0
\end{array}\right]\left(\bar{A}^{+} \bar{x}+K y\right),
$$

where $K$ is a basis matrix for the null-space of $\bar{A}$ and $y$ is an arbitrary vector. The latter can be written concisely as

$$
x(0)=\bar{K} y+x^{\prime}
$$

where $x^{\prime}:=\left[\begin{array}{ll}I_{n} & 0\end{array}\right] \bar{A}^{+} \bar{x}$ and $\bar{K}:=\left[\begin{array}{ll}I_{n} & 0\end{array}\right] K$. Now, we temporarily add to the original constraints (1) and (2), herein briefly denoted by $\mathcal{C}$, the additional constraint on the initial state $y=y_{0}$. This additional constraint sharply fixes the initial state of the resulting 
modified problem. Moreover, it is immediate to check that

$$
\inf _{\mathcal{C}} J(x, u)=\inf _{y_{0}}\left[\inf _{\mathcal{C}, y=y_{0}} J(x, u)\right]
$$

holds. The infimum inside square brackets on the right-hand side of (29) still has the form of Problem 1. Hence, we are concerned with the case of sharply assigned initial state. In this case the existence of solutions for Problem 1 is proved in [3, p. 85], where it is assumed that the matrix $S$ is zero. However, the general case when $S$ may differ from zero can be brought back to that of $S=0$ by assigning $u(t)=$ $-R^{-1} S^{T} x(t)+\omega(t)$ and by minimizing the quadratic index with respect to the new control variable $\omega(t)$. Thus, we get an equivalent problem where the corresponding matrix $S$ is zero. Hence, $\inf _{\mathcal{C}, y=y_{0}} J(x, u)$ is indeed a minimum. Moreover, in view of Theorem 3, such a minimum can be written as a positive semidefinite quadratic form in $\left[\begin{array}{c}y_{0} \\ k\end{array}\right]$ where $k:=\left[\begin{array}{c}w \\ z\end{array}\right]$. It follows that the infimum over $y_{0}$ on the right-hand side of (29) is a minimum as well. The existence of a minimum of $J(x, u)$ over the original constraints $\mathcal{C}$ guarantees that Problem 1 has solutions.

Now we are interested in proving an alternative necessary and sufficient condition for the existence of the optimal solution, which is given in terms of the problem data, so that it can be tested without the need of solving the algebraic Riccati and Lyapunov equations.

Corollary 1: Let $R_{0}$ be a basis matrix of the reachable subspace from the origin. Problem 1 admits solutions if and only if $w \in$ im $W Z$, where

$$
Z:=\left[\begin{array}{cc}
I_{n} & 0 \\
e^{A T} & R_{0}
\end{array}\right]
$$

Proof: By taking Theorem 4 into account, we will show that a trajectory satisfying (1) and (2) exists if and only if $w \in \operatorname{im} W Z$. Concerning the only if part, the existence of a trajectory satisfying (1) and (2) implies that a vector $\tau$ exists such that (27) holds, so that the constraint (2) can be written as

$$
W\left[\begin{array}{c}
x(0) \\
e^{A T} x(0)+R_{0} \tau
\end{array}\right]=W Z\left[\begin{array}{c}
x(0) \\
\tau
\end{array}\right]=w
$$

which clearly admits solutions if and only if $w \in \operatorname{im} W Z$. These steps can be performed in the reversed order, so as to prove the if part of the statement.

The following theorem addresses the issue of uniqueness of the solution of Problem 1. At first sight, one could think that a simple way to decide whether the solution of Problem 1 is unique is that of determining if $N \pi=\xi$ has a unique solution. When the pair $(A, B)$ is not reachable, however, there are cases when the solution of $N \pi=\xi$ is not unique, but Problem 1 admits only one solution. For example, consider the following matrices

$$
\begin{aligned}
& A=\left[\begin{array}{cc}
1 & 2 \\
0 & -1
\end{array}\right], \quad B=\left[\begin{array}{l}
2 \\
0
\end{array}\right], \\
& Q=I_{2}, \quad S=0, \quad R=1,
\end{aligned}
$$

with assigned initial and terminal states, that is, $x(0)=\left[\begin{array}{l}0 \\ 0\end{array}\right]$ and $x(T)=\left[\begin{array}{l}8 \\ 0\end{array}\right]$, where $T=2$. A simple computation shows that

$$
P_{u}=\left[\begin{array}{cc}
\frac{1+\sqrt{5}}{4} & \frac{1}{2} \\
\frac{1}{2} & 1
\end{array}\right] \text { and } Y=\left[\begin{array}{cc}
\frac{2 \sqrt{5}}{5} & 0 \\
0 & 0
\end{array}\right]
$$

are respectively an unmixed solution of the ARE and the corresponding solution of the closed-loop Lyapunov equation. Since the extreme states are both assigned, it follows that $W=I_{4}$ and $\xi=\left[\begin{array}{l}x(0) \\ x(T)\end{array}\right]$, so that

$$
N=W L=\left[\begin{array}{cccc}
1 & 0 & \frac{2 \sqrt{5}}{5} e^{-2 \sqrt{5}} & 0 \\
0 & 1 & 0 & 0 \\
e^{-2 \sqrt{5}} & 0 & \frac{2 \sqrt{5}}{5} & 0 \\
0 & e^{-2} & 0 & 0
\end{array}\right]
$$

which is clearly singular, and $\xi \in \operatorname{im} N$. Hence, the solutions of $N \pi=\xi$ are parameterized in the nullspace of $N$ as $\pi=N^{+} \xi+K_{N} v$, where $K_{N}=$ $\left[\begin{array}{llll}0 & 0 & 0 & 1\end{array}\right]^{\top}$ is a basis matrix of $\operatorname{ker} N$ and $v$ is an arbitrary vector. However, it can be easily checked that the optimal solution is unique, since, by taking (20) into account, a simple computation shows that

$$
\begin{gathered}
{\left[\begin{array}{ccc}
e^{A_{u} i} & Y e^{A_{u}^{\top}(T-t)} \\
-K_{u} e^{A_{u} t} & -R^{-1}\left(S^{\top} Y+B^{\top} P_{u} Y-B^{\top}\right) e^{A_{u}^{\top}(T-t)}
\end{array}\right] K_{N}} \\
=\left[\begin{array}{cccc}
e^{-\sqrt{5} t} & 0 & \frac{2 \sqrt{5}}{5} e^{-\sqrt{5}(2-t)} & 0 \\
0 & e^{-1} & 0 & 0 \\
\frac{1+\sqrt{5}}{2} e^{-\sqrt{5} t} & e^{-i} & \frac{5-\sqrt{5}}{5} e^{-\sqrt{5}(2-t)} & 0
\end{array}\right]\left[\begin{array}{l}
0 \\
0 \\
0 \\
1
\end{array}\right],
\end{gathered}
$$

which is equal to zero for any $t \in[0,2]$. Hence, all the solutions of $N \pi=\xi$ lead to the same optimal state and input functions for Problem 1. As a result, the uniqueness of the solutions of $N \pi=\xi$ is only a sufficient condition for the uniqueness of the solution of Problem 1. The following theorem, on the contrary, provides a necessary and sufficient condition 
depending only on the problem data; hence, it can be tested without the need of solving the ARE. As a consequence of this theorem, we obtain an alternative parameterization of all the solutions of Problem 1.

Theorem 5: Define $\tilde{Q}:=Q-S R^{-1} S^{\top}$ and $\tilde{A}:=$ $A-B R^{-1} S^{\top}$. Let

$$
\begin{gathered}
M:=\left[\begin{array}{c}
\Xi_{1} \\
\Xi_{2} \\
\Xi_{3}
\end{array}\right], \quad \text { where } \Xi_{1}:=\Theta\left[\begin{array}{c}
I_{n} \\
e^{\tilde{A} T}
\end{array}\right], \\
\Xi_{2}:=\left[\begin{array}{c}
\tilde{Q} \\
\tilde{Q} \tilde{A} \\
\vdots \\
\tilde{Q} \tilde{A}^{n-1}
\end{array}\right], \quad \Xi_{3}:=W\left[\begin{array}{c}
I_{n} \\
e^{\tilde{A} T}
\end{array}\right] .
\end{gathered}
$$

Suppose (1) and (2) admit solutions. A solution of Problem $l$ is unique if and only if $\operatorname{ker} M=\{0\}$.

Proof: (If). Let $x^{\prime}(t), u^{\prime}(t)$ and $x^{\prime \prime}(t), u^{\prime \prime}(t)$ be two different solutions of Problem 1. They both satisfy (5)(9) for suitable $\lambda^{\prime}(t), \nu^{\prime}, \lambda^{\prime \prime}(t), \nu^{\prime \prime}$, respectively. A straightforward computation over (5)-(9) shows that their difference $\quad x(t):=x^{\prime}(t)-x^{\prime \prime}(t), \quad u(t):=$ $u^{\prime}(t)-u^{\prime \prime}(t)$ satisfies (5)-(9) with $\lambda(t):=\lambda^{\prime}(t)-$ $\lambda^{\prime \prime}(t), \nu:=\nu^{\prime}-\nu^{\prime \prime}, z_{0}=z_{T}=0, w=0$, hence it is the optimal solution of the optimal control problem consisting of the minimization of $J(x, u)$ with $z_{0}=z_{T}=0$, under the constraints (1) and $W\left[\begin{array}{l}x(0) \\ x(T)\end{array}\right]=0$. For this problem, the identically zero state and input functions are optimal, since they satisfy all the constraints and the corresponding cost is zero. Hence, $x(t)$ and $u(t)$ are not identically zero but the corresponding cost is zero. In particular, this trajectory and the corresponding control $u(t)$ are such that

$$
\left[\begin{array}{cc}
Q & S \\
S^{T} & R
\end{array}\right]\left[\begin{array}{l}
x(t) \\
u(t)
\end{array}\right]=0 \quad \text { a.e. in }[0, T]
$$

since $\Pi=\Pi^{\top} \geq 0$. It follows that $u(t)=$ $-R^{-1} S^{\top} x(t)$, hence by substitution the optimal state trajectory $x(t)$ is a solution of the differential equation

$$
\dot{x}(t)=\left(A-B R^{-1} S^{\top}\right) x(t)=\tilde{A} x(t),
$$

and the integrand function of the performance index reduces to $x^{\top}(t) \tilde{Q} x(t)$, which is zero almost everywhere in $[0, T]$ if and only if $x(0) \in \operatorname{ker} \Xi_{2}$. From (31) it is found that $x(T)=e^{\tilde{A} T} x(0)$. Since $\Theta$ is positive semidefinite, the state trajectory $x(t)$ is optimal only if $\Theta\left[x^{\top}(0) \quad x^{\top}(T)\right]^{\top}=\Theta\left[x^{\top}(0) \quad x^{\top}(0) e^{\dot{A}^{\top} T}\right]^{\top}=0$, hence only if $x(0) \in \operatorname{ker} \Xi_{1}$. Finally, since $x(T)=$ $e^{\dot{A} T} x(0)$, from $W\left[\begin{array}{c}I_{n} \\ e^{A T}\end{array}\right] x(0)=0$, it follows that $x(0) \in \operatorname{ker} \Xi_{3}$. As a result, if the solution is not unique, a nonzero initial state $x(0)$ exists such that $x(0) \in \operatorname{ker} M$.

(Only if). Let $x^{0}(t)$ and $u^{\circ}(t)$ be an optimal state trajectory and the corresponding control law, respectively. Define

$$
\dot{\xi}(t)=\left(A-B R^{-1} S^{\top}\right) \xi(t)=\tilde{A} \xi(t),
$$

where $\xi(0)$ is supposed to be a nonnull vector of the null-space of $M$. Consider the new trajectories

$$
\begin{aligned}
& \bar{x}(t):=x^{o}(t)+\xi(t) \quad \text { and } \\
& \ddot{u}(t):=u^{o}(t)-R^{-1} S^{\top} \xi(t),
\end{aligned}
$$

We show that this state trajectory $\bar{x}(t)$, together with the input function $\bar{u}(t)$, is still optimal for Problem 1. First, notice that $\bar{x}(t)$ satisfies the constraints (2) since by definition $\xi(0) \in$ ker $\Xi_{3}$. Moreover,

$$
\begin{aligned}
& {\left[\begin{array}{c}
\tilde{x}(0)-z_{0} \\
\tilde{x}(T)-z_{T}
\end{array}\right]^{\top} \Theta\left[\begin{array}{c}
\tilde{x}(0)-z_{0} \\
\tilde{x}(T)-z_{T}
\end{array}\right]} \\
& =\left[\begin{array}{c}
x^{o}(0)-z_{0} \\
x^{o}(T)-z_{T}
\end{array}\right]^{\top} \Theta\left[\begin{array}{l}
x^{o}(0)-z_{0} \\
x^{o}(T)-z_{T}
\end{array}\right],
\end{aligned}
$$

since $\xi(0) \in \operatorname{ker} \Xi_{l}$. Finally,

$$
\begin{aligned}
& {\left[\begin{array}{ll}
x^{o}(t)^{\top} & u^{o}(t)^{\top}
\end{array}\right] \Pi\left[\begin{array}{l}
x^{o}(t) \\
u^{o}(t)
\end{array}\right]} \\
& =\left[\begin{array}{ll}
\bar{x}^{\top}(t) & \bar{u}^{\top}(t)
\end{array}\right] \Pi\left[\begin{array}{l}
\bar{x}(t) \\
\bar{u}(t)
\end{array}\right]
\end{aligned}
$$

since $\xi(0) \in \operatorname{ker} \Xi_{2}$. As a result, the two state trajectories $x^{\circ}(t)$ and $\bar{x}(t)$ are both optimal for Problem 1 .

Remark 5: Once given an optimal solution $x^{\mathrm{o}}(t)$, $u^{\circ}(t)$ for Problem 1, equations (32) and (33) provide an alternative parameterization of the set of optimal solutions of Problem 1 in terms of the set of initial states $x(0) \in \operatorname{ker} M$. In other words, (32) and (33) provide the expression of all the optimal solutions of Problem 1 - when the latter admits solutions - that are therefore defined modulo the free motions that originate from initial states belonging to the nullspace of $M$.

\section{The Riccati Differential Equation}

A further important consequence of Theorem 1 is the possibility of deriving an explicit expression for the 
solution of the Riccati differential problem with assigned terminal condition:

$$
\begin{aligned}
& \dot{P}(t)+A^{\top} P(t)+P(t) A \\
& -(P(t) B+S) R^{-1}\left(B^{\top} P(t)+S^{\top}\right)+Q=0, \\
& P(T)=P_{T} .
\end{aligned}
$$

First, we present a well-known result $[12$, pp. $274-$ 275], [7, p. 987] which relates the solution of (34) to that of the matrix Hamiltonian differential problem.

Lemma 3: Let $H$ be defined in (12). Let $T>0$ and $P_{T}=P_{T}^{\top} \geq 0$. Let $X, \Lambda:(-\infty, T] \rightarrow \mathbb{R}^{n \times n}$ be the solution of the matrix Hamiltonian differential problem

$$
\left[\begin{array}{c}
\dot{X}(t) \\
\dot{\Lambda}(t)
\end{array}\right]=H\left[\begin{array}{l}
X(t) \\
\Lambda(t)
\end{array}\right], \quad\left[\begin{array}{c}
X(T) \\
\Lambda(T)
\end{array}\right]=\left[\begin{array}{c}
I_{n} \\
P_{T}
\end{array}\right] .
$$

Then, $X(t)$ is nonsingular for all $t \in(-\infty, T]$ and the solution $P(t)$ of (34) equals $\Lambda(t) X^{-1}(t), t \in(-\infty, T]$.

Since Theorem 1 provides a closed-form representation of the solutions of (11), and hence of (35), it is not difficult to determine an explicit expression of $\Lambda(t) X^{-1}(t)$, as the following theorem explains. Similar formulae can be found in the literature, [7]. However, they are valid under more restrictive assumptions on the pair $(A, B)$, such as controllability or stabilizability.

Theorem 6: Let assumptions (Al)-(A2) hold. Let $P_{u}$ be a strongly unmixed solution of (14), let $A_{u}$ be given by (15) and $Y$ be the corresponding solution of (16). The matrix $P(t)=\Lambda(t) X^{-1}(t)$, with

$$
\begin{aligned}
X(t)= & e^{-A_{u}(T-t)}\left(I_{n}-Y\left(P_{u}-P_{T}\right)\right) \\
& +Y e^{A_{u}^{\top}(T-t)}\left(P_{u}-P_{T}\right), \\
\Lambda(t)= & P_{u} e^{-A_{u}(T-t)}\left(I_{n}-Y\left(P_{u}-P_{T}\right)\right) \\
& +\left(P_{u} Y-I_{n}\right) e^{A_{u}^{\top}(T-t)}\left(P_{u}-P_{T}\right),
\end{aligned}
$$

is the solution of (34).

Proof: As a straightforward consequence of Theorem 1, the solutions of the differential equation (35) can be parameterized in $\Phi, \Psi \in \mathbb{R}^{n \times n}$ as

$$
\left[\begin{array}{l}
X(t) \\
\Lambda(t)
\end{array}\right]=\left[\begin{array}{c}
I_{n} \\
P_{u}
\end{array}\right] e^{A_{u} t} \Phi+\left[\begin{array}{c}
Y \\
P_{u} Y-I_{n}
\end{array}\right] e^{A_{u}^{\mathrm{T}}(T-t)} \Psi
$$

By imposing the terminal conditions $X(T)=I_{n}$ and $\Lambda(T)=P_{T}$ on the latter, we find $\Phi=e^{-A_{u} T}\left(Y P_{T^{-}}\right.$ $\left.Y P_{u}+I_{n}\right)$ and $\Psi=P_{u}-P_{T}$. Thus, the corresponding $X(t)$ and $\Lambda(t)$ are then given by (36) and (37). Hence, by virtue of Lemma $3, X(t)$ is invertible for all $t \in$ $(-\infty, T]$ and $P(t)=\Lambda(t) X^{-1}(t)$ is the solution of (34).

\section{Stabilizable Pairs}

If the pair $(A, B)$ is stabilizable, in Theorem 1 we can choose as a strongly unmixed solution of the ARE (14) the maximal solution $P_{+}=P_{+}^{\top} \geq 0$ that is stabilizing, that is, such that all the eigenvalues of the corresponding closed-loop matrix $A_{+}$are in the open left-half complex plane, $[21$, p. 354]. In this case, the expressions of the optimal state and input functions are given in terms of the matrix exponentials $\exp [A+t]$ and $\exp \left[A_{+}^{\top}(T-t)\right]$. Hence, the optimal solution involves exponentials of strictly stable matrices in the overall time interval $[0, T]$, thus ensuring that its computation is numerically robust even for large time horizons. Furthermore, in this case the matrices $P_{+}$, $A_{+}$and $Y$ may be computed by standard and reliable algorithms available in any control package (see e.g. the MATLAB ${ }^{\circledR}$ routines care.m and lyap.m).

\section{1. $\mathrm{H}_{2}$ Decoupling with Preview}

An interesting example of a possible application of the approach presented in Section 3 is the $H_{2}$ decoupling of previewed input signals. The $\mathrm{H}_{2}$ decoupling by state feedback is a well-known and deeply investigated method for the rejection of an unknown input, [14,24]. When the signal to be decoupled is known in advance, a great improvement can be achieved in its rejection by taking advantage of this pre-knowledge through a feedforward unit whose input is the previewed input signal, and that accounts for the so-called pre-action, $[28,15,27,20]$. Consider the LTI system $\Sigma$

$$
\dot{x}(t)=A x(t)+B_{1} u(t)+B_{2} w(t), \quad x(0)=0,
$$

$$
y(t)=C x(t)+D_{1} u(t)+D_{2} w(t)
$$

where, for all $t \geq 0, w(t) \in \mathbb{R}^{r}$ is the input signal to be decoupled from the output $y(t) \in \mathbb{R}^{p}$. The signal $w(t)$ is supposed to be zero in $[0, T)$ and known in advance with a preview time $T>0$. Define the previewed input $w_{p}(t):=w(t+T), t \geq 0$.

Problem 2: Find an LTI feedforward compensator $\Sigma_{c}$ connected as in Fig. 2 such that the transfer function matrix $\hat{G}(s)$ of the overall system $\hat{\Sigma}$, from the previewed input $w_{p}(t)$ to the output $y(t)$, is internally stable and its $\mathrm{H}_{2}$ norm $\|\hat{G}\|_{2}$ is minimized. 


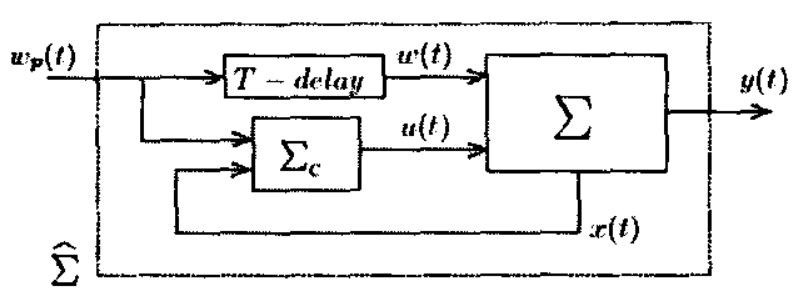

Fig. 2. $H_{2}$-optimal decoupling scheme of a previewed signal $w_{p}$.

Let $\left(e_{i}\right)_{i \in\{1, \ldots, r\}}$ be the standard basis of $\mathbb{R}^{r}$. Recall that the $\mathrm{H}_{2}$ norm of $\hat{G}(s)$ can be expressed in terms of the impulse response matrix $\hat{G}(t)$ of $\hat{\Sigma}$ as

$$
\|\hat{G}\|_{2}^{2}=\sum_{i=1}^{r} \int_{0}^{\infty} \hat{\mathcal{G}}_{i}^{\top}(t) \hat{\mathcal{G}_{i}}(t) d t
$$

where $\hat{\mathcal{G}}(t)$ denotes the $i$-th column of $\hat{\mathcal{G}}(t)$, that is, is the response of $\hat{\Sigma}$ with zero initial state to the input $w_{p}(t)=e_{i} \delta(t),(\delta(t)$ denoting the Dirac impulse), [14, p. 265]. Assume that

(B1) the pair $\left(A, B_{1}\right)$ is stabilizable;

(B2) $\left(A, B_{1}, C, D_{1}\right)$ has no invariant zeros on $t \mathbb{R}$ and $D_{1}$ is full-column rank;

(B3) $\operatorname{im} D_{2} \subseteq \operatorname{im} D_{1}$.

Define $Q:=C^{\top} C, S:=C^{\top} D_{1}$ and $R:=D_{1}^{\top} D_{1}$. Assumption (B2) ensures that $R=R^{\top}>0$ and that the corresponding Hamiltonian matrix (12) has no eigenvalues on $\mathbb{R},[29$, Theorem 13.7, Lemma 13.9, Corollary 13.10]. Hence, assumptions (B1)-(B2) ensure that the stabilizing solution $P_{+}=P_{+}^{\top} \geq 0$ of the ARE (14), with $B:=B_{1}$, exists.

Theorem 7: Consider Problem 2. Let assumptions (B1)-(B3) hold. Let $P_{+}=P_{+}^{\top} \geq 0$ be the stabilizing solution of the $A R E$ (14), let $A_{+}=A-B_{1} K_{+}$, where $K_{+}:=R^{-1}\left(S^{\top}+B_{1}^{\top} P_{+}\right)$. The optimal compensator $\Sigma_{c}$ for Problem 2 is described by the following inputoutput relation:

$$
\begin{aligned}
u(t)= & \int_{0}^{T} \Phi(\tau) w_{p}(t-\tau) d \tau-K_{+} x(t) \\
& -D_{1}^{+} D_{2} w(t)
\end{aligned}
$$

where $\Phi(t):=-R^{-1} B_{1}^{\top} e^{A_{+}^{\top}(T-t)} P_{+} B_{2}$.

Proof: First, suppose $D_{0}=0$. Let $i \in\{1, \ldots, r\}$. Consider the problem of minimizing the $i$-th term of the sum in (40). As already observed, this is equivalent to finding $u_{i}(t), t \in[0,+\infty)$, that minimizes $\int_{0}^{\infty} y_{i}^{\mathrm{T}}(t) y_{i}(t) d t$, where $y_{i}(t)$ is the output of (38)-(39) where $w_{i}(t)=e_{i} \delta(t-T)$, whose effect is the instantaneous transition of the state $x_{i}(t)$ at $t=T$ :

$$
x_{i}(T)=\int_{0}^{T} e^{A(T-\tau)}\left(B_{1} u_{i}(\tau)+B_{2} e_{i} \delta(\tau-T)\right) d \tau .
$$

Hence, $x_{i}(T)=B_{2}^{i}+x_{i}\left(T_{-}\right)$, where $B_{2}^{i}$ is the $i$-th column of $B_{2}$ and $x_{i}\left(T_{-}\right):=\int_{0}^{T} e^{A(T-\tau)} B_{1} u_{i}(\tau) d \tau$ is the state at $t=T$ obtained by the application of the sole input $u_{i}(t), t \in[0, T)$. The optimal control $u_{i}(t)$, $t \in[T,+\infty)$, is then obtained by solving an infinitehorizon LQ problem consisting of the minimization of the quadratic index $\int_{T}^{\infty} y_{i}^{\mathrm{T}}(t) y_{i}(t) d t$ with initial condition $x_{i}(T)$, and can be expressed by the algebraic state feedback $u_{i}(t)=-K_{+} x_{i}(t)=-K_{+} e^{A_{+}(t-T)} x_{i}(T)$, $t \geq T$. The corresponding optimal cost is given by the quadratic form $x_{i}^{\top}(T) P_{+} x_{i}(T)$, [17, Theorem 16.3.3]. Hence, the contribution of the infinite-horizon part can be expressed by the end-point penalty term $x_{i}^{\top}(T) P_{+} x_{i}(T)=\left(x_{i}\left(T_{-}\right)+B_{2}^{i}\right)^{\top} P_{+}\left(x_{i}\left(T_{-}\right)+B_{2}^{i}\right)$, which is added to the finite-horizon part. Hence, the optimal control law $u_{i}(t), t \in[0, T)$, is the solution of the minimization of the functional

$$
\begin{aligned}
J_{i}:= & \int_{0}^{T} y_{i}^{\top}(t) y_{i}(t) d t \\
& +\left(x_{i}\left(T_{-}\right)+B_{2}^{i}\right)^{\top} P_{+}\left(x_{i}\left(T_{-}\right)+B_{2}^{i}\right) .
\end{aligned}
$$

The minimization of this index can be achieved as a particular case of Problem 1, in which $\Theta_{1}=\Theta_{2}=0$, $\Theta_{3}=P_{+}, z_{T}=-B_{2}^{i}$ and $W=\left[\begin{array}{ll}I_{n} & 0\end{array}\right]$. The optimal state and costate can be expressed by (17) in terms of $p$ and $q$, where $P_{u}=P_{+}$and $A_{u}=A_{+}$. The boundary conditions $x_{i}(0)=0$ and $\lambda_{i}(T)=P_{+}\left(x_{i}(T)+B_{2}^{i}\right)$ derived from (6) and (8) yield

$$
p_{i}=Y e^{A_{+}^{\top} T} P_{+} B_{2}^{i} \text { and } q_{i}=-P_{+} B_{2}^{i} \text {, }
$$

where $Y$ is the solution of (16) with $B=B_{1}$. By virtue of (10), the optimal $u_{i}(t), t \in[0, T)$ is obtained by replacing (44) in

$$
u_{i}(t)=-K_{+} e^{A_{+} t} p_{i}-\left(K_{+} Y-R^{-1} B_{1}^{\top}\right) e^{A_{+}^{\top}(T-t)} q_{i} .
$$

On the other hand, as already observed, for $t \geq T$ the optimal control law is $u_{i}(t)=-K_{+} e^{A_{+}(t-T)} x_{i}(T)$, where, by virtue of (42), (17) and (44) we find $x_{i}(T)=$ $B_{2}^{i}+e^{A_{+} T} p_{i}+Y q_{i}$. It is easily checked that the control scheme in the statement, which does not depend on $i$, gives rise to this input function when fed by the input $w_{i}(t)=e_{i} \delta(t-T)$, so that it minimizes each $J_{i}$ simultaneously. 
If $D_{2}$ differs from zero, Problem 2 can still be solved, provided that the condition $\operatorname{im} D_{2} \subseteq \operatorname{im} D_{1}$ is satisfied. In fact, by taking (39) into account we easily see that the impulse response matrix $\hat{\mathcal{G}}(t)$ would involve a term $D_{2} \delta(t-T)$ such that the integral in (40) would diverge, unless its contribution is directly canceled by a part of the control input $u(t)$. In other words, the $\mathrm{H}_{2}$ norm of the overall system is finite if and only if $\operatorname{im} D_{2} \subseteq \operatorname{im} D_{1}$ and the control input has the following structure:

$$
u(t)=-D_{1}^{+} D_{2} w(t)+u_{c}(t)
$$

where the first part $-D_{1}^{+} D_{2} w(t)$ cancels the feedthrough term $D_{2} w(t)$ from the output since $\operatorname{im} D_{2} \subseteq \operatorname{im} D_{1}$, while the term $u_{c}(t)$ is the control that follows from the procedure presented as Theorem 7 . In fact, we have recast the problem as one of the types dealt with in Theorem 7, that is, in which the feedthrough matrix from $w(t)$ to $y(t)$ is zero, where $u(t)$ is now replaced by $u_{c}(t)$ and $B_{2} w(t)$ is replaced by $\left(B_{2}-B_{1} D_{1}^{+} D_{2}\right) w(t)$.

The inner structure of the optimal compensator $\Sigma_{c}$ involves a finite impulse response system, whose impulse response matrix is $\Phi(t)$ for $t \in[0, T)$ and zero elsewhere, an algebraic state feedback $-K_{+} x(t)$ and a feedforward term $-D_{1}^{+} D_{2} w(t)$. The transfer function matrix $G_{F I R}(s)$ of the finite impulse response system is given by the $\mathcal{L}$-transform of $\Phi(t)$, that leads to

$$
\begin{aligned}
G_{F I R}(s)= & R^{-1} B_{1}^{\top} e^{A_{+}^{\top} T}\left(A_{+}^{\top}+s I_{n}\right)^{-1} \\
& \times\left(e^{-\left(A_{+}^{\top}+s I_{n}\right) T}-I_{n}\right) P_{+} B_{2}
\end{aligned}
$$

\section{An Example and Concluding Remarks}

In this section, we present a simple example of $\mathrm{H}_{2}$ decoupling with preview, and we briefly discuss the results presented in this paper.

\subsection{An Illustrative Example}

Consider a system (38)-(39), where

$$
A=\left[\begin{array}{cccc}
0.5 & 1 & -0.4 & 0 \\
0.1 & 0.7 & 0 & -0.5 \\
0 & 0 & 0.4 & 0 \\
0 & 0 & 0 & 0.6
\end{array}\right]
$$

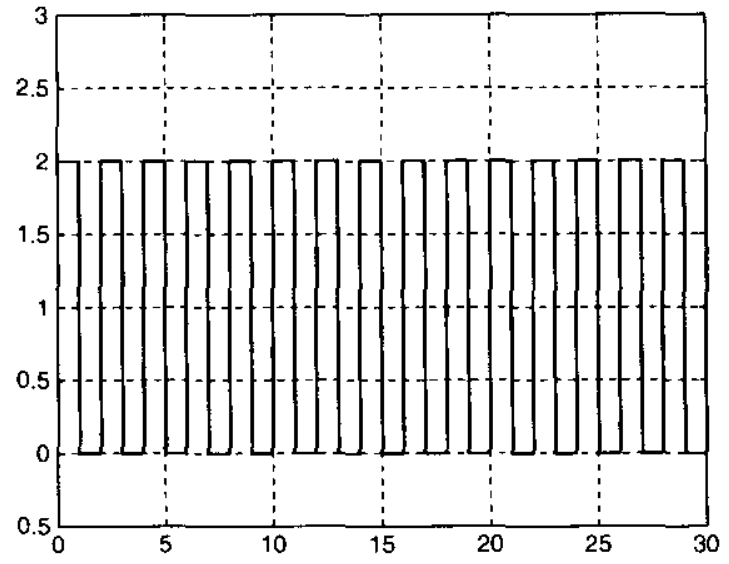

Fig. 3. Input function $w(t)$.

$$
\begin{aligned}
B_{1} & =\left[\begin{array}{ll}
1 & 0 \\
0 & 1 \\
1 & 0 \\
0 & 1
\end{array}\right], \quad B_{2}=\left[\begin{array}{l}
1 \\
0 \\
0 \\
3
\end{array}\right], \\
C & =\left[\begin{array}{llll}
1 & 0 & 0 & 0 \\
0 & 1 & 0 & 0
\end{array}\right], \quad D_{1}=\left[\begin{array}{cc}
1.2 & 0.3 \\
-0.1 & 0.8
\end{array}\right], \\
D_{2} & =\left[\begin{array}{l}
2 \\
3
\end{array}\right],
\end{aligned}
$$

subject to the scalar input $w(t)$ depicted in Fig. 3, which is a clock-type function with values in $\{0,2\}$ and duty cycle 0.5 . By following the design procedure outlined in Section 6.1, aimed at deriving a compensator $\Sigma_{c}$ for the rejection of the previewed signal $w(t)$ from the output $y(t)$ of $\Sigma$, in Fig. 4 we compare the different output functions that are obtained by varying the value of the preview time $T$.

In fact, in this case, assumptions (B1)-(B3) hold true. The optimal compensator $\Sigma_{c}$ can be designed as shown in Theorem 7, working jointly with the algebraic unit accounting for the feedforward action $-D_{1}^{+} D_{2} w(t)$. The first subplot in Fig. 4 shows the rejection that can be achieved when the preview time $T$ is zero, that is, when the signal $w(t)$ to be decoupled is accessible for measurement but not previewed. The second and the third subplots present the output $y(t)$ for increasing values of $T$, that is, for $T=1$ and $T=4$. As we could expect, the rejection achieved considerably improves as the preview interval increases. Note also that in this interval there is an evident transient, which is due to the fact that the input function $w(t)$, in the case considered, is not zero in $[0, T]$. Hence, during this transient, the compensator 

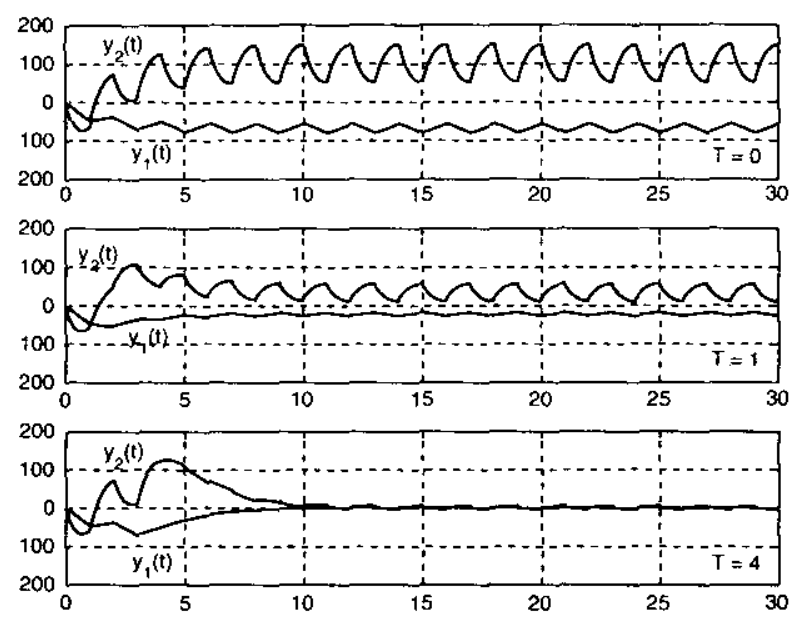

Fig. 4. Output functions obtained with the preview time $T=0$, $T=1$ and $T=4$, respectively.

has no previewed information on the actual value of $w$ $(t)$, and no rejection is possible except for that determined by the feedforward term $-D_{1}^{+} D_{2} w(t)$. Even if all the outputs in Fig. 4 seem to be equal in $[0, T]$, this is not true: during this time interval the compensator takes into account the future information on $w$ $(t)$, so as to minimize the effect of $w(t)$ on $y(t)$ when $t>T$.

\subsection{Conclusions}

In this paper, a generalized version of the finitehorizon LQ problem has been presented and solved. The method proposed for its solution, based on a parameterization of the state-costate functions satisfying the Hamiltonian differential equation, presents several advantages.

- It is based only on algebraic tools, that is, the ARE and the Lyapunov equation. Thus, it does not require the integration of the differential Riccati equation (which is well-known to be very demanding from a computational viewpoint).

- It can be applied under the very weak assumption of sign-controllability of $(A, B)$.

- It yields a useful formula for the optimal cost, as a quadratic form of the problem data. The closedform expression of the optimal trajectory and of the optimal cost can be exploited for the analysis of the structural properties of the optimal solutions and for solving more general (possibly parametric) optimization problems, for example, problems having the $L Q$ as a subproblem. For example, consider the case of two (or more) optimal control problems that are coupled by constraints or by a weight on the respective boundary conditions.

- In the case of stabilizability of the underlying system, the numerical robustness of the present solution is ensured if we choose as a strongly unmixed solution of the ARE its maximal solution $P_{+}$, that can be very easily determined by widespread and reliable software routines. Moreover, in this case the parameterization, and consequently the computation of the parameters $p$ and $q$ associated with the optimal solution appear to be robust, since the matrix exponentials in (17) are well-conditioned for all $t \in[0, T]$.

- The application of this methodology for the determination of a closed-form formula of the solution of the differential Riccati equation with assigned terminal condition has been briefly discussed in Section 5. Finally, an easy solution has been proposed to the $\mathrm{H}_{2}$ decoupling with preview, based on the previous results.

\section{References}

1. Abou-Kandil H, Freiling G, Ionescu V, Jank G. Matrix Riccati equations in control and systems theory. Birkhaser, Boston, 2003

2. Athans M, Falb PL. Optimal control: an introduction to the theory and its applications. McGraw-Hill, New York, 1966

3. Berkovitz LD. Optimal control theory. Springer-Verlag, New York, 1974

4. Bolzern P, Colaneri P, De Nicolao G. On discrete-time $\mathbf{H}_{\infty}$ fixed-lag smoothing. IEEE Trans Signal Process, 2004; 52: 132-141

5. Bolzern P, Colaneri P, De Nicolao G, Shaked U. Guaranteed $\mathrm{H}_{\infty}$ bounds for Wiener filtering and prediction. Int $\mathrm{J}$ Robust Nonlinear Control, 2002; 12(1): 41-56

6. Brunovsky P, Komomik J. The Riccati equation solution of the linear quadratic problem with constrained terminal state. IEEE Trans Autom Control, 1981; AC-26(2): 398-402

7. Callier FM, Winkin J, Willems JL. Convergence of the time-invariant Riccati differential equation and LQ-problem: mechanisms of attraction. Int J Control, 1994; 59(4): 983-1000

8. Colaneri P, Ferrante A. A J-spectral factorization approach for $\mathrm{H}_{\infty}$ estimation problems in discrete-time. IEEE Trans Autom Control, 2002; AC-47(12): 2108-2113

9. Colaneri P, Ferrante A. Algebraic Riccati equation and Jspectral factorization in $\mathbf{H}_{\infty}$ estimation. Syst Control Lett, 2004; 41(5): 383-393

10. Colaneri P, Ferrante A. Algebraic Riccati equation and Jspectral factorization for $\mathrm{H}_{\infty}$ smoothing and deconvolution. SIAM J Control and Optim, 2006; 45: 123-145

11. Colaneri P, Maroni $M$, Shaked U. $\mathrm{H}_{\infty}$ prediction and smoothing for discrete-time systems: a J-spectral factorization approach. In Proceedings of the 37th IEEE Conference on Decision and Control (CDC 1998), Tampa, USA, December 1998; 3: pp. 2836-2842 
12. Coppel WA. Linear-quadratic optimal control. In Proceedings of the Royal Society of Edinburgh, 1974; 73A: pp. 271-289

13. Ferrante A, Marro G, Ntogramatzidis L. A parametrization of the solutions of the finite-horizon LQ problem with general cost and boundary conditions. Automatica, 2005; 1 (8): $1359-1366$

14. Ionescu V, Oara C, Weiss M. Generalized Riccati theory and robust control, a Popov function approach. Wiley, 1999

15. Kojima A, Ishijima S. LQ preview synthesis: optimal control and worst case analysis. IEEE Trans Autom Control, 1999; AC-44(2): 352-357

16. Kučera V. Algebraic Riccati equations: Hermitian and definite solutions. In S. Bittanti et al. (Eds.), The Riccati Equation, Communications and Control Engeneering, Springer, Berlin, 1991

17. Lancaster $P$, Rodman L. Algebraic Riccati equations. Clarendon Press, Oxford, 1995

18. Lewis FL, Syrmos V. Optimal Control. John Wiley \& Sons, New York, 1995

19. Mangasarian OL. Sufficient conditions for the optimal control of nonlinear systems. SIAM J Control, 1966; 4(1): $139-152$

20. Marro G, Ntogramatzidis L, Zattoni E. $\mathrm{H}_{2}$-Optimal Decoupling of Previwed Signals in the Continuous-Time Domain. In Proceedings of the 2004 American Control Conference
(2004 ACC), Boston, Massachussets (USA), June 30-July 2 2004. pp. 2717-2722

21. Molinari BP. The time-invariant linear-quadratic optimal control problem. Automatica, 1977; 13:347-357

22. Ntogramatzidis L. A unified approach to the Linear Quadratic Regulator with applications to $\mathrm{H}_{2}$ problems with delays. PhD thesis, University of Bologna, 2005

23. Perez H, Devasia S. Optimal output-transitions for linear systems. Automatica, 2003; 39(2): 181-192

24. Saberi A, Sannuti P, Chen BM. $\mathrm{H}_{2}$ Optimal Control. System and Control Engineering. Prentice Hall International, London, 1995

25. Scherer $C$. The solution set of the algebraic Riccati equation and the algebraic Riccati inequality. Linear Algebr Appl, 1991; 153: 99-122

26. Shayman MA. Geometry of the algebraic Riccati equation, Part I. SIAM J Control Optim, 1983; 21: 375-394

27. Tadmor G, Mirkin L. $\mathrm{H}_{\infty}$ control and estimation with preview-part I: matrix ARE solutions in continuous time. IEEE Trans Autom Control, 2005; AC-50(1): $19-28$

28. Tomizuka M. Optimal continuous finite preview problem. IEEE Trans Autom Control, 1975; AC-20(3): 362365

29. Zhou K, Doyle J, Glover K. Robust and Optimal Control. Prentice Hall, New York, 1996 